\title{
Morphometric analysis of feedforward pathways from the primary somatosensory area (S1) of rats
}

\author{
A.L. de Sá ${ }^{1}$, C.P. Bahia ${ }^{2}$, V.C. Correa ${ }^{3}$, I.A. Dias ${ }^{2}$, C. Batista ${ }^{2}$, W. Gomes-Leal ${ }^{3}$, A.L.S. Pinho ${ }^{4}$ \\ J.C. Houzel ${ }^{5}$, C.W. Picanço-Diniz ${ }^{6}$ and A. Pereira ${ }^{1}$ \\ ${ }^{1}$ Instituto do Cérebro, Universidade Federal do Rio Grande do Norte, Natal, RN, Brasil \\ ${ }^{2}$ Laboratório de Neuroplasticidade, Instituto de Ciências da Saúde, Universidade Federal do Pará, Belém, PA, Brasil \\ ${ }^{3}$ Laboratório de Neuroproteção e Neurorregeneração Experimental, Instituto de Ciências Biológicas, \\ Universidade Federal do Pará, Belém, PA, Brasil \\ ${ }^{4}$ Departamento de Estatística, Universidade Federal do Rio Grande do Norte, Natal, RN, Brasil \\ ${ }^{5}$ Laboratório de Fronteiras em Neurociências, Instituto de Ciências Biomédicas, Universidade Federal do Rio de Janeiro, \\ Rio de Janeiro, RJ, Brasil \\ ${ }^{6}$ Laboratório de Investigações em Neurodegeneração e Infecção, Instituto de Ciências Biológicas, Universidade Federal do Pará, \\ Belém, PA, Brasil
}

\begin{abstract}
We used biotinylated dextran amine (BDA) to anterogradely label individual axons projecting from primary somatosensory cortex (S1) to four different cortical areas in rats. A major goal was to determine whether axon terminals in these target areas shared morphometric similarities based on the shape of individual terminal arbors and the density of two bouton types: en passant $(\mathrm{Bp})$ and terminaux $(\mathrm{Bt})$. Evidence from tridimensional reconstructions of isolated axon terminal fragments ( $\mathrm{n}=111)$ did support a degree of morphological heterogeneity establishing two broad groups of axon terminals. Morphological parameters associated with the complexity of terminal arbors and the proportion of beaded Bp vs stalked Bt were found to differ significantly in these two groups following a discriminant function statistical analysis across axon fragments. Interestingly, both groups occurred in all four target areas, possibly consistent with a commonality of presynaptic processing of tactile information. These findings lay the ground for additional work aiming to investigate synaptic function at the single bouton level and see how this might be associated with emerging properties in postsynaptic targets.
\end{abstract}

Key words: Somatosensory cortex; Barrel field; Feedforward networks; Axon terminal; Axon morphometry

\section{Introduction}

Nocturnal rodents such as rats and mice rely on whisker contacts with external objects to gather information from their peri-individual space (1). Tactile inputs from the whiskers are transduced by mechanoreceptors and make synaptic connection in the brainstem trigeminal nuclei and the thalamus before reaching the cortex (for review, see 2). At least three parallel pathways carry ascending tactile information to the cortex: the lemniscal, extralemniscal and paralemniscal pathways, which are relayed by distinct regions in the thalamus, the ventral posteromedial nucleus in the lemniscal pathway and the posterior medial nucleus in the extralemniscal and paralemniscal pathway, respectively $(3,4)$.

The main target of thalamocortical axons is the primary somatosensory area (S1), which in rodents is arranged cytoarchitectonically in two divisions: a granular zone characterized by dense cell aggregates in layer IV called barrels, and a cell-sparse dysgranular zone comprised by septa and other regions surrounding the barrel field $(3,5)$. Even though barrels are also present in regions representing other body parts in $S 1$ (6), barrels associated with the whiskers are larger and have a distinct isomorphic arrangement in the posteromedial barrel subfield (PMBSF) resembling the spatial distribution of whiskers on the snout (5).

From S1, somatosensory information goes through several additional processing stages in higher-order areas. This processing is not strictly hierarchical, given that many feedback projections intervene in the process (7). From S1, information is sent simultaneously to the secondary somatosensory area (S2), the parietal ventral area (PV), the parietal rhinal area (PR), and the contralateral S1 (S1c) (8-13) where it is integrated spatiotemporally (14). Similar to S1, areas S2 and PV are also organized topographically, with a complete representation of the contralateral body, and

Correspondence: A. Pereira: <pereira@neuro.ufrn.br> 
also receive direct thalamocortical inputs $(15,16)$. PR, on the other hand, receives projections from S2 and PV, but does not have a well-defined topographical organization. PR is located in the posterior insula and receives auditory and somatosensory inputs in rats (17).

Some studies have shown that morphological attributes of axon terminals, such as the size of terminals (18), are associated with different functional roles in neuronal pathways (19). However, even though there is ample evidence of parallel processing within modality-specific sensory channels (e.g., 20), there are few examples of morphologically distinct types of axon terminals. For instance, glutamatergic corticocortical pathways in rodents are classified into two types, called class 1 and class 2, based on terminal morphology (21-23).

We used anterograde neuronal tracer injections to compare the morphology of feedforward axon's terminal fragments projections from $\mathrm{S} 1$ to higher order somatosensory areas. Our aim was to compare the morphology of these pathways and contribute to the understanding of their role in somatosensory processing. Our results suggest that information from S1 reach its targets through two parallel pathways. In a step towards classification, we present evidence for differences in the density of two types of boutons, terminaux and en passant, in feedforward projections from S1.

\section{Material and Methods}

Male adult Wistar rats (300-350 g; $n=8)$ were obtained from the Central Animal Facility of the Universidade Federal do Pará (UFPA), Brazil. Experimental procedures followed the Guide for the Care and Use of Laboratory Animals (NIH publication, No. 86-23, revised 1985) and were approved by the UFPA's Ethics Committee for the Use of Animals (BIO015-09). All efforts were made to reduce the number of animals used and to avoid suffering.

One day before surgery, rats were premedicated with dexamethasone $(1.0 \mathrm{mg} / \mathrm{kg}, \mathrm{im})$ to prevent brain edema and with vitamin $\mathrm{K}(1.0 \mathrm{mg} / \mathrm{kg}, \mathrm{im})$ to avoid excessive bleeding during surgery. Immediately before surgery, animals received a dose of atropine sulfate $(0.1 \mathrm{mg} / \mathrm{kg}, \mathrm{im})$ and anesthesia was induced with ketamine $(100 \mathrm{mg} / \mathrm{kg}, \mathrm{im})$ and xylazine $(5 \mathrm{mg} / \mathrm{kg}, \mathrm{im})$. If necessary, supplementary doses of ketamine (100 $\mathrm{mg} / \mathrm{kg}$, im) were provided during the surgical procedure. Body temperature was maintained at about $37^{\circ} \mathrm{C}$ with the aid of a heating pad (Harvard Bioscience Co., USA).

\section{Surgical procedures and tracer injection}

The head of the animal was secured in a stereotaxic apparatus (David Kopf, Germany) and a single burr hole was made at the stereotaxic coordinates AP -2.0, ML $5.0 \mathrm{~mm}$, corresponding to the PMBSF in S1. Then, the dura mater was punctured and a single iontophoretic injection of $10 \%$ biotinylated dextran amine $10 \mathrm{KD}$ (BDA, Molecular Probes, USA) diluted in saline phosphate buffer
(PBS, $\mathrm{pH} 7.4,0.1 \mathrm{M}$ ) was made through a glass capillary (20-30 $\mu \mathrm{m}$ internal tip diameter) by applying $5 \mu \mathrm{A}$ positive current pulses (7s ON, 7s OFF) over 3-5 min using a current source (Stoelting Co, USA). We aimed for this procedure to be consistently reproducible in order to guarantee a reasonable degree of representativeness for the labeled terminals originating from that specific region. The animals were allowed to recover in their own cages with food and water ad libitum. After 15 days, they were anesthetized with an overdose of ketamine (24) and perfused transcardially with PBS followed by $4 \%$ paraformaldehyde in phosphate buffer $(\mathrm{PB}, \mathrm{pH} 7.4$, $0.1 \mathrm{M})$. The brains were removed from the skull and cut with a vibratome (Pelco, USA) into serial, 150- $\mu \mathrm{m}$ thick coronal sections. Sections were washed three times, 20 min each, in PB and once in a solution of $3 \%$ Triton $\mathrm{X}-100$ in $\mathrm{PB}$, before being incubated overnight, freefloating in the avidin/biotin/peroxidase complex (ABC, 1:200; Vector Laboratories, USA) at room temperature under constant agitation. Peroxidase labeling was revealed using the diaminobenzidine reaction intensified with nickel ammonium sulfate (25). Finally, sections were dehydrated in rising alcohol concentrations, cleared in xylene and coverslipped with Entellan (Merck, Germany). After reconstruction, the sections were processed with Nissl staining to allow delineation of cortical.

\section{Morphometry}

For each animal, all consecutive sections were first checked for the absence of retrogradely labeled cells located distant from the immediate vicinity of the injection site. Labeled axons arising from the injection site were then examined at both low and high magnification. Individual axons were followed up to their entry into the grey matter, and individual terminal branches arborizing into target cortical areas were finally selected for computer-assisted $3 \mathrm{D}$ reconstruction on the basis of the following criteria: absence of branching points previous to entry in the target cortex (with the exception of the cut end of the thicker parental branch) and the entire arbor of the axon terminal should appear to be contained within a single thick section. In order to reduce sampling bias, only 1-5 terminal branches were selected per area in each animal (Table 1). After selection, well-labeled axon terminal fragments in S2 $(n=25), \operatorname{PV}(n=27), \operatorname{PR}(n=31)$, and S1c $(n=28)$ were reconstructed directly from coronal sections using a $60 \times$ oil immersion objective installed on an Optiphot-2 microscope (NIKON, Japan) equipped with a high-resolution Lucivid micromonitor (MBF Bioscience, USA) attached to a drawing tube and a 3D-motorized stage MAC5000 (Ludl, USA). After, all 3D-reconstructed sections were stained with the Nissl method to reveal the architecture of cortical layers. The devices were connected to a desktop computer running the Neurolucida software (MBF Bioscience, USA), thereby allowing for the 
Table 1. Number of primary somatosensory area (S1) feedforward fragments axon terminals to secondary somatosensory area $(\mathrm{S} 2)$, parietal ventral area (PV), parietal rhinal area (PR) and contralateral primary somatosensory area (S1c) reconstructed from each case of neuronal tract tracing $\mathrm{S} 1$ microinjections in a total of $n=8$ animals.

\begin{tabular}{lcccc}
\hline Animal ID (subtotal) & S2 & PV & PR & S1c \\
\hline $1(17)$ & 6 & 2 & 5 & 4 \\
$2(15)$ & 4 & 3 & 4 & 4 \\
$3(18)$ & 4 & 4 & 5 & 5 \\
$4(13)$ & 3 & 5 & 1 & 4 \\
$5(10)$ & 2 & 3 & 3 & 2 \\
$6(13)$ & 2 & 3 & 6 & 2 \\
$7(11)$ & 2 & 3 & 3 & 3 \\
$8(14)$ & 2 & 4 & 4 & 4 \\
Total (111) & 25 & 27 & 31 & 28 \\
\hline
\end{tabular}

recording and analysis of $\mathrm{x}, \mathrm{y}$, and $\mathrm{z}$ coordinates of digitized points. Photomicrographs were taken with a digital camera attached to the microscope; image brightness and contrast were adjusted offline with Adobe Photoshop (Adobe Systems, USA).

\section{Statistical analysis}

The following morphometric parameters of axon terminal fragments were analyzed in ipsilateral S1, S2, PV, PR, and contralateral S1c: density of en passant boutons per millimeter (number of $\mathrm{Bp}$ per millimeter, Bpd), density of terminaux boutons per millimeter (number of $\mathrm{Bt}$ per millimeter, Btd), total density of boutons per millimeter (BTd: Bpd plus Btd), density of branching points (number of bifurcations per millimeter), density of segments (number of segments per millimeter), average length (total length per segment), index of Bp (number of Bp divided by the total number of boutons) and index of $\mathrm{Bt}$ (number of Bt divided by
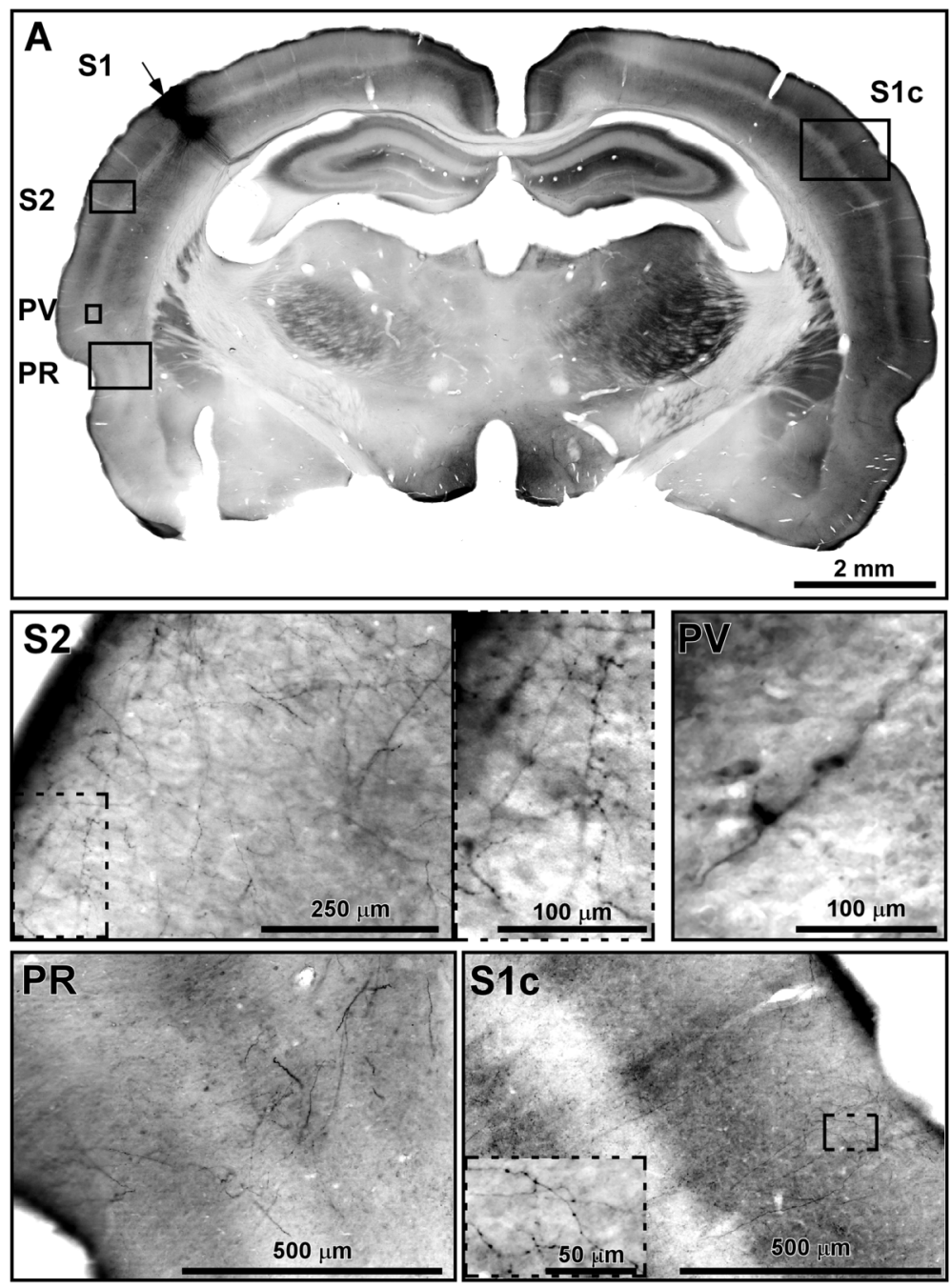

Figure 1. Photomicrographs of anterograde labeling of corticocortical axons after a single iontophoretic injection of BDA in S1. A, Lowpower photomontage $(\times 20$ objective virtual slide) of an entire coronal section showing the location of a typical injection site in area S1 (arrow) and of target areas containing anterogradely labeled axons. Below, photomicrographs with variable magnifications illustrating anterogradely-labeled axons originating in $\mathrm{S} 1$ and terminating within the second somatosensory area (S2), the parietal-ventral area (PV), the parietal-rhinal area (PR), and the contralateral S1 (S1c). Insets show branches with boutons terminaux and en passant. Scale bars are indicated for each panel. 
the total number of boutons). We did not correct for tissue shrinkage, since our study was eminently comparative and based on parameters not affected by shrinkage. To assess the homogeneity of the axonal population in each area in relation to defined morphometric variables, we first performed a multivariate analysis of variance (MANOVA). Next, an exploratory cluster analysis of morphological terminal types was performed using hierarchical clustering analysis (HCA) to explore whether specific groups of terminals existed in our sample based on the morphometric variables mentioned above (26-28). The significance of the classification performed by the HCA was tested with MANOVA. Based on the resulting classification, discriminant analysis was used to identify the variables that contributed most strongly to the separation. Average values for morphometric parameters are reported as means $\pm S E$ and compared across different groups using analysis of variance (ANOVA) and the Tukey post hoc test, with $\alpha=0.05$.

\section{Technical considerations}

One possible methodological issue is the fact that axon terminals represent only a fragment of the parental axon arbor and it is possible that different reconstructed fragments may originate from the same parental axon. While this possibility would not affect the morphological appearance of the fragments, it could introduce some bias in the study since larger axonal arbors may have more labeled terminals and therefore a higher probability to contribute to the sample. We tried to offset this bias by reconstructing only one terminal fragment per histological section for each target area, as shown in Table 1.

\section{Results}

Morphological analysis was based on a sample of 111 BDA-labeled terminal fragments (see Table 1). All BDA iontophoretic injections were confined to $\mathrm{S} 1$ and exhibited a dense black central core, ranging from 300-500 $\mu \mathrm{m}$ in diameter, surrounded by anterogradely-labeled cell bodies and axonal fragments belonging to intracortical circuits and spanning layers II to VI (Figure 1). Cortical layers could be discerned on sections counterstained with Nissl (Figure 2) due to the faint background staining from the diffuse peroxidase activity under lower magnification (Figure 1). Only layer I was not shown because it was poorly labeled and couldn't be well discerned as can be
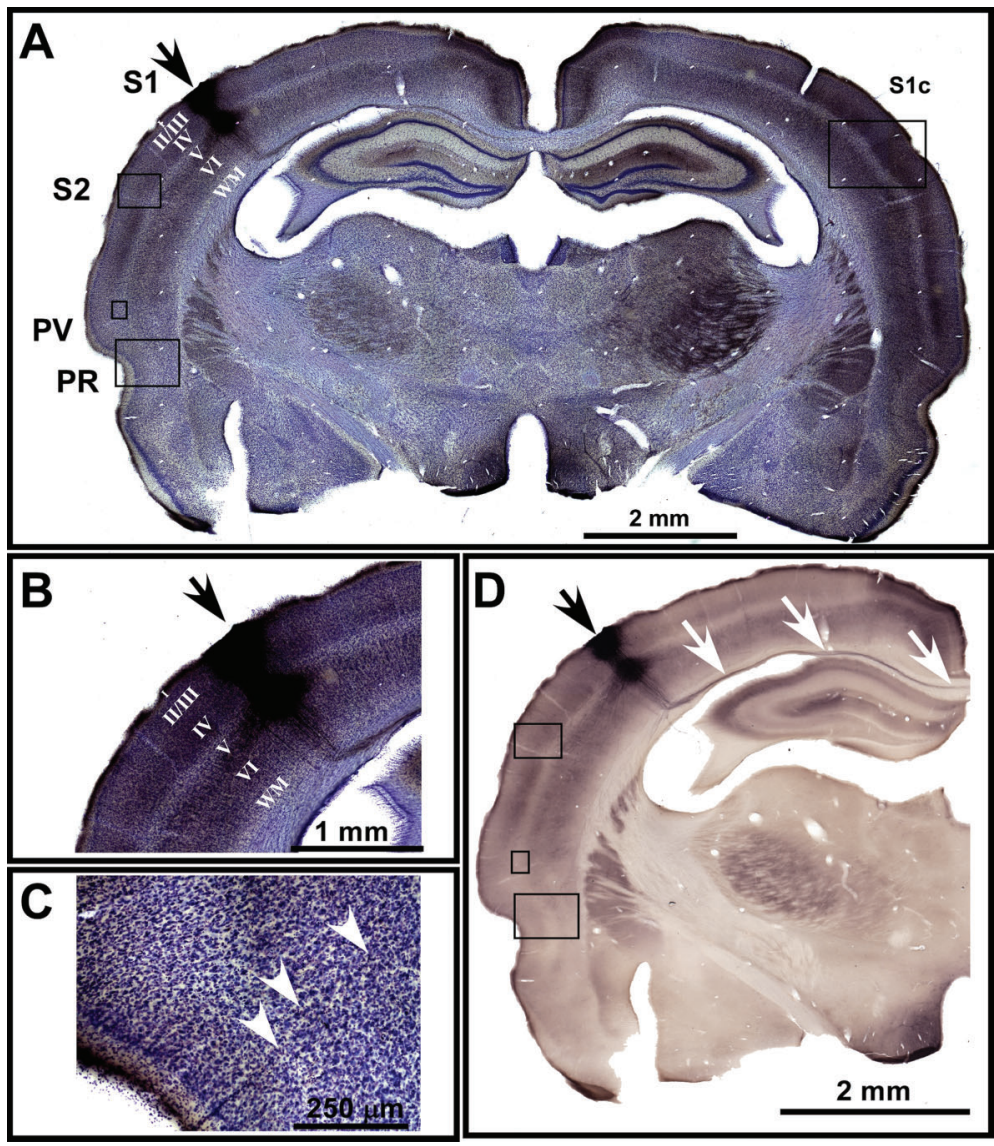

Figure 2. Histological rat brain section doublestained with Nissl showing the laminar location of labeled corticocortical axon terminals after a single iontophoretic injection of biotinylated dextran amine in S1. A, Low-power photomontage of the same coronal section depicted in Figure 1. $B$, Photomicrograph showing cortical layers and $C$, anterogradely-labeled axon terminals in a section double-stained with Nissl. Arrows indicate axon terminal segments. $D$, Black arrow indicates injection site and white arrows indicate callosal axons. The boxes indicate cortical areas (S2, PV and PR) where axon fragments labeled with DBA were reconstructed. Cortical layers are indicated by roman numerals, from I to $\mathrm{Vl}$; WM: white matter. 
seen in Figure $2 \mathrm{~A}$ and $\mathrm{B}$. We did not find retrogradely labeled cell bodies outside the immediate vicinity of the iontophoretic injection sites. Labeled axons could be followed from $\mathrm{S} 1$ to target regions located in ipsilateral S2, PV, and PR areas. Callosal axon terminals were also found in homotopic regions in the contralateral $S 1$. Terminal axon segments bearing boutons were located in all cortical layers, except layer I (Figures 1 and 2). This is in accordance with their feedforward nature (29). We could identify both $\mathrm{Bp}$ and $\mathrm{Bt}$ boutons studding from axon fragments labeled with BDA. The former can be associated with a thickening of the axon, while the latter resemble spiny appendages located at the endings of axonal branches.

The average morphometric parameter values for axon fragments located in S1 targets were as follows: segment length per millimeter (S2=0.12 $\pm 0.01 ; P V=0.15 \pm 0.02$; $P R=0.12 \pm 0.01 ; S 1 c 0.16 \pm 0.01)$, segment density per millimeter $(S 2=10.47 \pm 1.0 ; P V=9.31 \pm 1.3 ; P R=9.66 \pm 0.6$; $\mathrm{S} 1 \mathrm{c}=9.06 \pm 0.9)$, number of branching points per millimeter $(S 2=5.64 \pm 0.50 ; P V=5.41 \pm 0.53 ; P R=5.77 \pm 0.62$; $\mathrm{S} 1 \mathrm{C}=5.23 \pm 0.59)$, and total bouton density per millimeter (Bp plus Bt; S2=67.54 $\pm 7.9 ; \mathrm{PV}=60.00 \pm 8.7 ; \mathrm{PR}=49.51 \pm$ 5.8; $\mathrm{S} 1 \mathrm{c}=65.18 \pm 7.4$ ).

MANOVA analysis did not demonstrate any significant segregation of morphometric variables, according to target area. Thus, axon fragments located in S2, PV, PR, and S1c seem to comprise a homogeneous population characterized by strong morphological similarities (Wilks test: $F=1.1122, P=0.3393$; Hotelling-Lawley test: $F=1.1145$, $P=0.3367)$.

ANOVA demonstrated significant differences $(F=1.2$; $\mathrm{P} \leqslant 0.05)$ in the relative number of $\mathrm{Bp}$ and $\mathrm{Bt}$ within individual cortical areas (Figure 3). Interestingly, the total density of boutons appeared to be similar in all target areas (Figure $3 \mathrm{~B}$ ). This may suggest that the synaptic efficacy of these pathways is similar in those areas. This is different from intracortical circuit connections, for instance, where connectivity is a function of spatial separation between neurons (30).

The discriminant analysis confirmed the separation of the data in two morphologically distinct groups and also revealed which variables were most important for classification (Figure 4). The discriminant analysis produced two linear functions having weight coefficients for each morphometric variable. The equations for $\mathrm{S} 2$ are as follows: $\mathrm{Y} 1=$ $-0.1195 \times \mathrm{X} 1-0.1352 \times \mathrm{X} 2-0.3881 \times \mathrm{X} 3+0.9038 \times \mathrm{X} 4$ and $Y 2=0.1630 \times X 1 \quad-0.0036 \times X 2 \quad-0.1392 \times X 3+$ $0.9768 \times \mathrm{X} 4$. It is worth mentioning that dimension $\mathrm{Y} 1$ is already sufficient to separate terminal groups in $\mathrm{S} 2$, as can be seen in Figure 4, where group I is associated with values smaller than group II. The same pattern is replicated for the remaining targets: $\mathrm{PV}(\mathrm{Y} 1=-0.0245 \times \mathrm{X} 1-0.1637 \times \mathrm{X} 2$ $-0.4955 \times \mathrm{X} 3+0.8527 \times \mathrm{X} 4$ and $\mathrm{Y} 2=0.0163 \times \mathrm{X} 1$ $-0.0572 \times \mathrm{X} 2+0.2295 \times \mathrm{X} 3+0.9715 \times \mathrm{X} 4), \mathrm{PR}(\mathrm{Y} 1=$ $-0.0104 \times \mathrm{X} 1-0.2576 \times \mathrm{X} 2-0.3650 \times \mathrm{X} 3+0.8946 \times \mathrm{X} 4$ and $\mathrm{Y} 2=0.9666 \times \mathrm{X} 1+0.0257 \times \mathrm{X} 2-0.0471 \times \mathrm{X} 3+$ $0.2476 \times \mathrm{X} 4)$, and S1c $(\mathrm{Y} 1=-0.2408 \times \mathrm{X} 1-0.2290 \times \mathrm{X} 2$ $-0.5083 \times \mathrm{X} 3+0.7945 \times \mathrm{X} 4$ and $\mathrm{Y} 2=-0.2498 \times \mathrm{X} 1$ $-0.0089 \times \mathrm{X} 2+0.3284 \times \mathrm{X} 3+0.9083 \times \mathrm{X} 4)$.

Figure 5 shows the dendograms obtained with the HCA performed on data from terminals located in the four target areas. The terminals from each area are identified at the bottom of the graphs and merge into discrete clusters at distinct stages, depending on their degree of morphological similarity. The dendograms suggested the
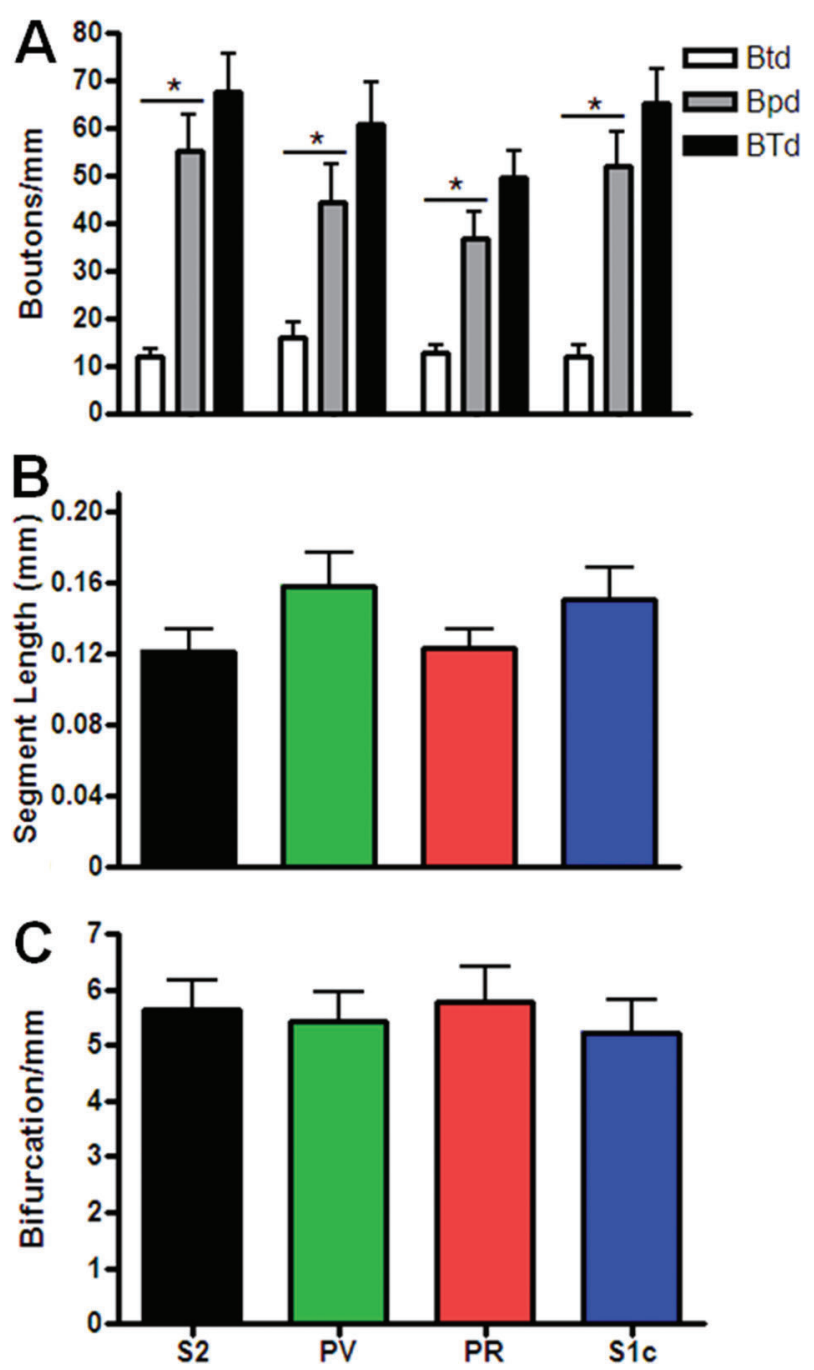

Figure 3. Multivariate discriminant statistical analysis shows that the linear density per millimeter of en passant boutons (Bpd) was higher than that of terminaux boutons (Btd) ( ${ }^{*} \mathrm{P}<0.05$, Bpd vs Btd; ANOVA-Tukey post hoc test) in all cortical target areas $(A)$. BTd: $\mathrm{Bpd}+\mathrm{Btd}$. Axon terminals in secondary somatosensory area $(\mathrm{S} 2)$, parietal rhinal area (PR), parietal ventral area (PV) and contralateral primary somatosensory area (S1C) presented similar morphological components $(P>0.05)$ based on segment length per millimeter $(B)$ or bifurcation density per millimeter $(C)$. 

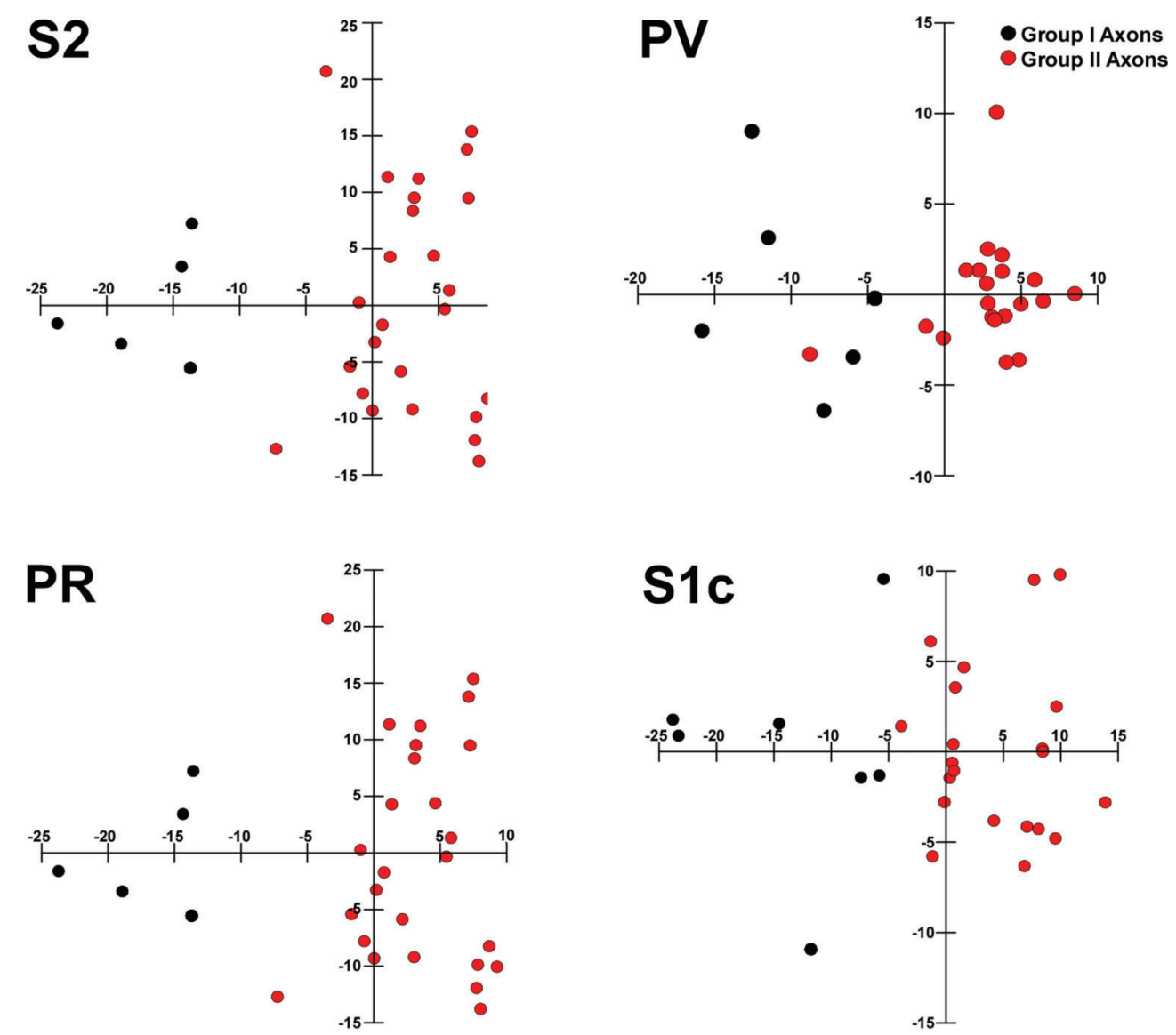

Figure 4. Graphic results of discriminant analysis showing the separation of axon terminals from secondary somatosensory area (S2), parietal rhinal area (PR), parietal ventral area (PV) and contralateral primary somatosensory area (S1c) into two distinct groups: Group I axons (black dots) and Group II Axons (red dots).

existence of two morphologically distinct groups of terminals (group I and group II) in each one of the studied areas (Figure 5) (ANOVA $F=1.0 ; P<0.01$ ).

Axon fragments from group I displayed a higher density of $\mathrm{Bp}(\mathrm{S} 2=100 \pm 21, \mathrm{PV}=86 \pm 9, \mathrm{PR}=96 \pm 8$ and $\mathrm{S} 1 \mathrm{c}=$ $102 \pm 1.20)$ and $B t(S 2=13 \pm 9, P V=11 \pm 5, P R=11 \pm 2$ and $\mathrm{S} 1 \mathrm{c}=15 \pm 7)$ than group II fragments $-\mathrm{Bp}(\mathrm{S} 2=30 \pm 16$, $P V=25 \pm 3, P R=25 \pm 3$ and $S 1 c=36 \pm 4)$ and $B t(S 2=11 \pm 7$, $P V=16 \pm 3, P R=13 \pm 1$ and $S 1 c=11 \pm 1$ ), (ANOVA $F=1.0$; $P<0.01$; Figure 6).

\section{Discussion}

The morphometric analysis of axon terminal arbors filled with BDA revealed that corticocortical projections originating in $\mathrm{S} 1$ and targeting somatosensory areas in both hemispheres seem to be morphologically similar. According to our results, despite this similarity, intrinsically associated with the density of two types of presynaptic boutons (beaded en passant boutons and stalked terminaux boutons) and the geometry of terminal arbors, the HCA suggested the presence of more than one group of terminals. This was further confirmed by MANOVA and by discriminant analysis (Figures 5 and 6 ).

Regarding the differences on the relative density of Bt and $\mathrm{Bp}$ between the two terminal groups, the question is whether there is any functional correlation associated with this finding. Both types of boutons have been structurally associated with synapses $(31,32)$. Even though the precise relationship between form and function in this case is still not determined, it has been proposed that Bt could be more involved with presynaptic facilitation and show more structural plasticity than $\mathrm{Bp}$, due to more efficient calcium storage (33). The findings from De Paola (34) suggest the possibility of a difference in the plastic potential between the two terminal groups we found in this work.

In rodents, tactile information from the whiskers is conveyed to $S 1$ by at least 3 pathways $(3,4,34,35)$. These pathways have been implicated with carrying information about distinct whisking attributes and remain relatively segregated in S1 (35). However, very little is known about 

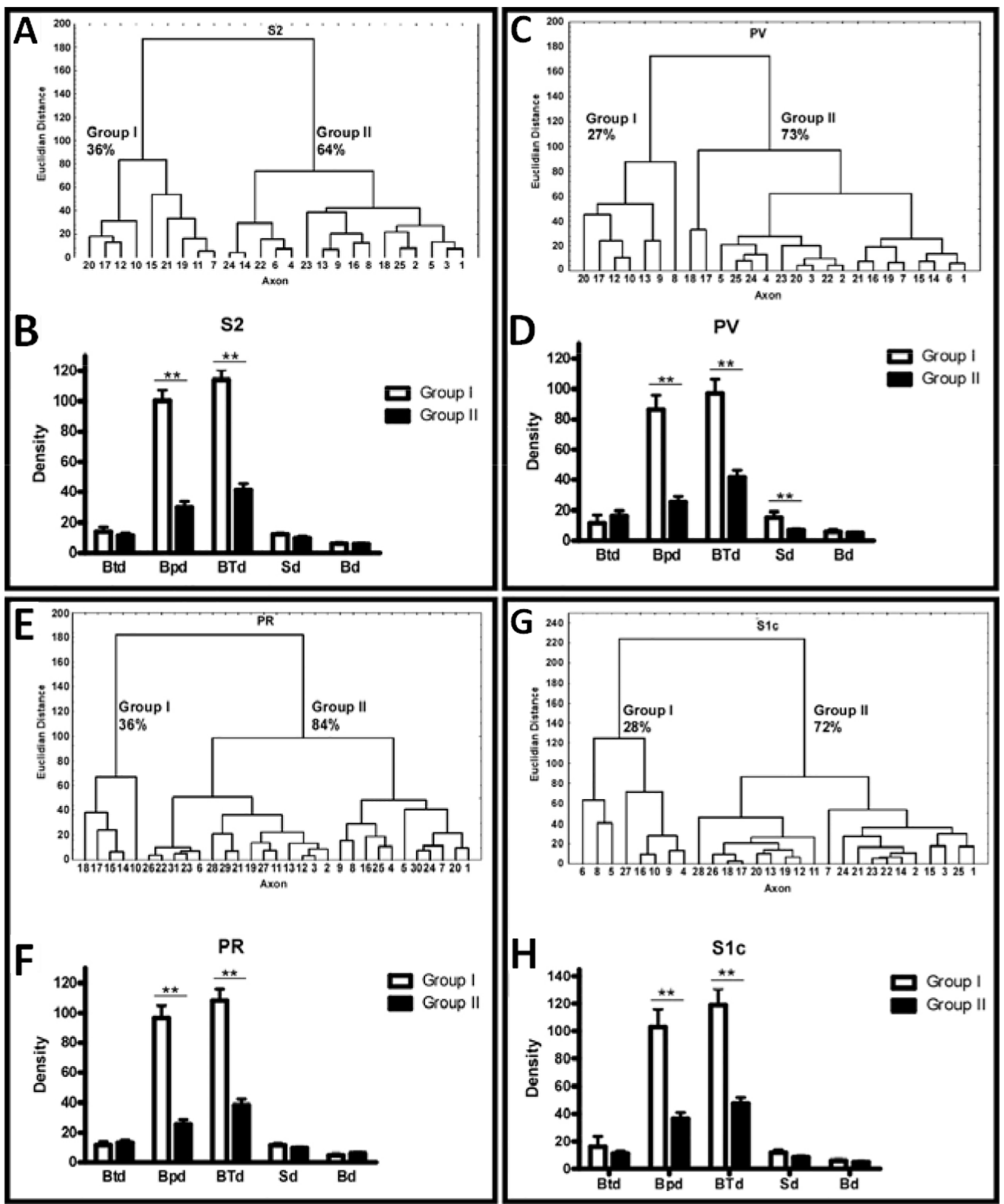

Figure 5. Hierarchical cluster analysis (HCA) dendrogram showing that feedforward axon terminals from $\mathrm{S} 1$ can be separated into two groups (I and II) in secondary somatosensory area (S2) $(A, B)$, parietal ventral area $(\mathrm{PV})(C, D)$, parietal rhinal area $(\mathrm{PR})(E, F)$ and contralateral primary somatosensory area $(\mathrm{S} 1 \mathrm{c})(G, H)$. The variable that most contributed to the distinction between groups was the density of boutons terminaux (Btd) $\left({ }^{*} \mathrm{P}<0.05\right.$, ANOVA-Tukey post hoc test). Sd: segment density per millimeter; Bpd: en passant boutons; Bd: bifurcation density per millimeter; BTd: Bpd + Btd 


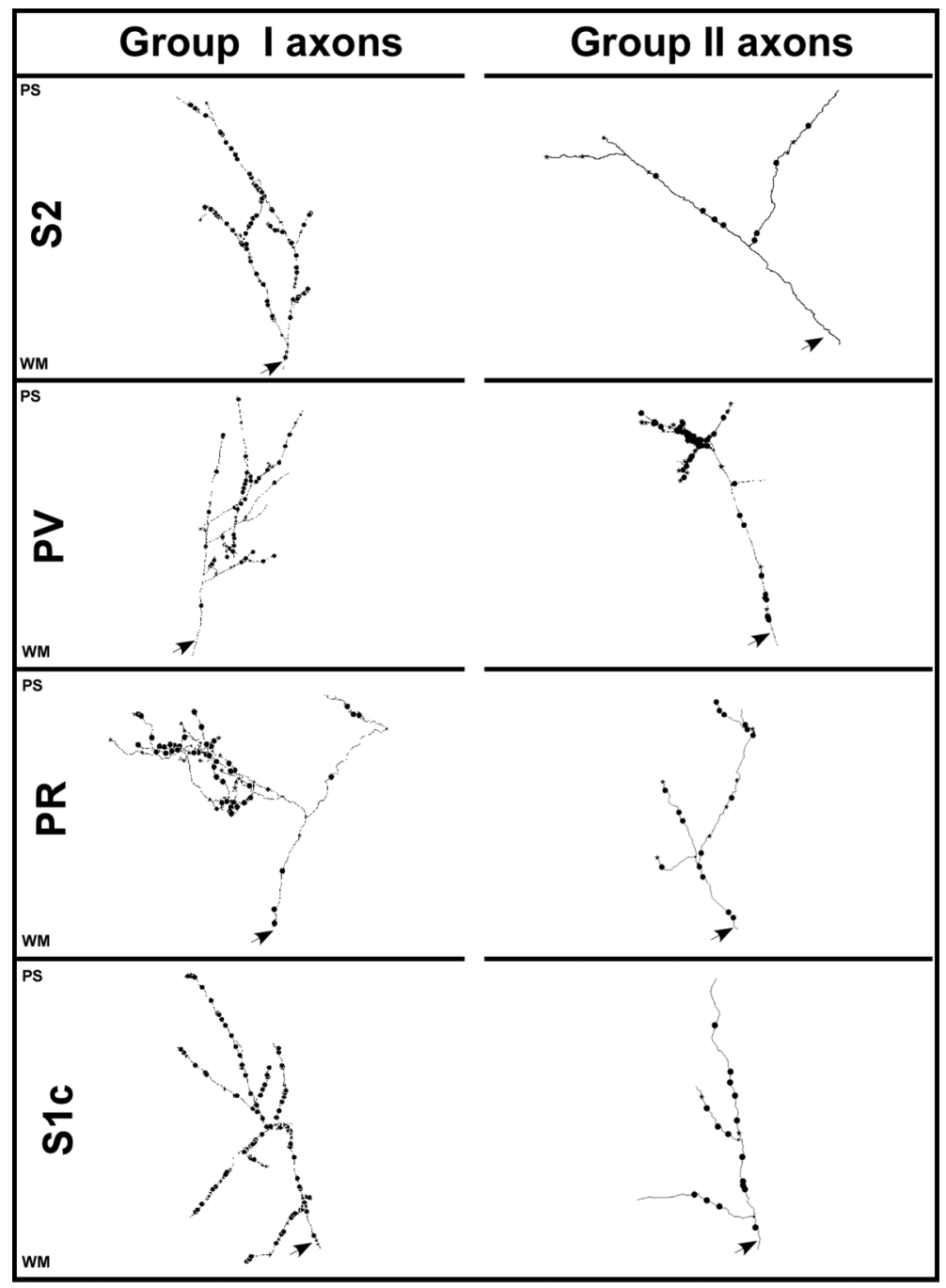

Figure 6. Representative examples of digitally reconstructed axon terminals in secondary somatosensory area (S2), parietal rhinal area $(\mathrm{PR})$, parietal ventral area (PV) and contralateral primary somatosensory area (S1c). Terminals are separated according to their profile into groups I and II. The relative position of both the pia mater and white matter borders are depicted in the figure. Arrows indicate the parent axon branch. PS: pial surface; WM: white matter.

their relative contribution to feedforward projections from S1 to S2, PV, PR, S1c. In the cortex, based on studies on synaptic properties and anatomical features, Sherman and Guillery (23) reported that glutamatergic projections can be classified into Class 1 and Class 2, depending on their role as circuit drivers or modulators, respectively. This separation between the driving and modulatory functions of glutamatergic projections can also be seen in the somatosensory pathways mentioned above and that carry information from the whiskers to $\mathrm{S} 1$, through synaptic relays in the trigeminal nuclei to the thalamus (16). The study by Viaene and coworkers (16) suggests that the role of the paralemniscal pathway is to provide modulatory inputs to S1, while the lemniscal pathway conveys precise information about whisker deflections to $\mathrm{S} 1$ and plays a role in object localization and identification (35). The modulatory role of the paralemniscal pathway (16) is also under the influence of the locus coeruleus (36). The most conspicuous morphological difference between drive and modulator pathways lies on the size and shape of boutons, with smaller Bt associated with driving connections and larger ones associated with a modulatory role (37).

While the size of Bp might affect axon dynamics, it is reasonable to suppose that such dynamics can also be affected later by other structural aspects of axon terminals, such as the relative distribution of $\mathrm{Bp}$ and $\mathrm{Bt}$ (33). As discussed above, Bt can probably facilitate synaptic potentials (33) in an activity-dependent manner (38). Such dynamic control of synaptic sensitivity could increase both the sensitivity and fidelity of transmission of sensory signals along driving pathways. Bp, on the other hand, could have a more modulatory effect, extending the functional reach of lemniscal and paralemniscal pathways 
beyond S1. The differences in the profile of group I and II terminals can also affect their susceptibility to plasticity, as evidenced by studies in the adult visual cortex of both rodents and primates $(39,40)$ showing that the turnover rate of $\mathrm{Bt}$ is significantly higher than $\mathrm{Bp}(40)$.

Our results suggest that feedforward projections from $\mathrm{S} 1$ are sent to at least 4 other cortical regions, including contralateral S1, in the rat. Morphologically, these inputs are very similar and can be further subdivided into two classes of terminals. Other studies had previously presented evidence for the existence of two categories of glutamatergic terminals in corticocortical pathways in

\section{References}

1. Grant RA, Mitchinson B, Prescott TJ. The development of whisker control in rats in relation to locomotion. Dev Psychobiol 2012; 54: 151-168, doi: 10.1002/dev.20591.

2. Petersen $\mathrm{CC}$. The functional organization of the barrel cortex. Neuron 2007; 56: 339-355, doi: 10.1016/j.neuron.2007. 09.017.

3. Alloway KD. Information processing streams in rodent barrel cortex: the differential functions of barrel and septal circuits. Cereb Cortex 2008; 18: 979-989, doi: 10.1093/cercor/ bhm138.

4. Pierret $\mathrm{T}$, Lavallee $\mathrm{P}$, Deschenes $\mathrm{M}$. Parallel streams for the relay of vibrissal information through thalamic barreloids. $J$ Neurosci 2000; 20: 7455-7462.

5. Woolsey TA, Van der Loos $\mathrm{H}$. The structural organization of layer IV in the somatosensory region (SI) of mouse cerebral cortex. The description of a cortical field composed of discrete cytoarchitectonic units. Brain Res 1970; 17: 205242, doi: 10.1016/0006-8993(70)90079-X.

6. Wallace MN. Histochemical demonstration of sensory maps in the rat and mouse cerebral cortex. Brain Res 1987; 418: 178-182, doi: 10.1016/0006-8993(87)90977-2.

7. Cooke DF, Goldring AB, Baldwin MK, Recanzone GH, Chen A, Pan T, et al. Reversible deactivation of higher-order posterior parietal areas. I. Alterations of receptive field characteristics in early stages of neocortical processing. $J$ Neurophysiol 2014; 112: 2529-2544, doi: 10.1152/jn.00140.2014.

8. Aronoff R, Matyas F, Mateo C, Ciron C, Schneider B, Petersen CC. Long-range connectivity of mouse primary somatosensory barrel cortex. Eur J Neurosci 2010; 31: 2221-2233, doi: 10.1111/j.1460-9568.2010.07264.x.

9. Disbrow E, Roberts T, Krubitzer L. Somatotopic organization of cortical fields in the lateral sulcus of Homo sapiens: evidence for SII and PV. J Comp Neurol 2000; 418: 1-21, doi: 10.1002/(SICI)1096-9861(20000228)418:1 < 1::AID-CNE1 > 3.0.CO;2-P.

10. Krubitzer L, Clarey J, Tweedale R, Elston G, Calford M. A redefinition of somatosensory areas in the lateral sulcus of macaque monkeys. J Neurosci 1995; 15: 3821-3839.

11. Krubitzer LA, Kaas JH. The organization and connections of somatosensory cortex in marmosets. J Neurosci 1990; 10: 952-974.

12. Remple MS, Henry EC, Catania KC. Organization of somatosensory cortex in the laboratory rat (Rattus norvegicus): Evidence for two lateral areas joined at the representation of the visual cortex. In the visual cortex, these two groups of terminals can be differentiated in terms of the functional role they play in their targets (driving or modulatory). In the somatosensory cortex, they could provide a substrate for the continuing segregation of parallel pathways beyond $\mathrm{S} 1$.

\section{Acknowledgments}

This study was financially supported by $\mathrm{CNPq}$ (\#476627/2011-7, \#483404/2013-6 and \#447835/2014-9) and CAPES-Cofecub (819-14).

the teeth. J Comp Neurol 2003; 467: 105-118, doi: 10.1002/ cne.10909.

13. Santiago LF, Rocha EG, Freire MA, Dias IA, Lent R, Houzel $\mathrm{JC}$, et al. The organizational variability of the rodent somatosensory cortex. Rev Neurosci 2007; 18: 283-294, doi: 10.1515/REVNEURO.2007.18.3-4.283.

14. Zhu Z, Disbrow EA, Zumer JM, McGonigle DJ, Nagarajan SS. Spatiotemporal integration of tactile information in human somatosensory cortex. BMC Neurosci 2007; 8: 21, doi: 10.1186/1471-2202-8-21.

15. Liang M, Mouraux A, lannetti GD. Parallel processing of nociceptive and non-nociceptive somatosensory information in the human primary and secondary somatosensory cortices: evidence from dynamic causal modeling of functional magnetic resonance imaging data. J Neurosci 2011; 31: 8976-8985, doi: 10.1523/JNEUROSCI.6207-10.2011.

16. Viaene AN, Petrof I, Sherman SM. Properties of the thalamic projection from the posterior medial nucleus to primary and secondary somatosensory cortices in the mouse. Proc Natl Acad Sci U S A 2011; 108: 18156-18161, doi: 10.1073/ pnas. 1114828108 .

17. Rodgers KM, Benison AM, Klein A, Barth DS. Auditory, somatosensory, and multisensory insular cortex in the rat. Cereb Cortex 2008; 18: 2941-2951, doi: 10.1093/cercor/ bhn054.

18. Li J, Wang S, Bickford ME. Comparison of the ultrastructure of cortical and retinal terminals in the rat dorsal lateral geniculate and lateral posterior nuclei. J Comp Neurol 2003; 460: 394-409, doi: 10.1002/cne.10646.

19. Rouiller EM, Welker E. A comparative analysis of the morphology of corticothalamic projections in mammals. Brain Res Bull 2000; 53: 727-741, doi: 10.1016/S03619230(00)00364-6.

20. Nassi JJ, Callaway EM. Parallel processing strategies of the primate visual system. Nat Rev Neurosci 2009; 10: 360372, doi: 10.1038/nrn2619.

21. Hoogland PV, Welker E, Van der Loos H. Organization of the projections from barrel cortex to thalamus in mice studied with Phaseolus vulgaris-leucoagglutinin and HRP. Exp Brain Res 1987; 68: 73-87, doi: 10.1007/BF00255235.

22. Hoogland PV, Wouterlood FG, Welker E, Van der Loos H. Ultrastructure of giant and small thalamic terminals of cortical origin: a study of the projections from the barrel cortex in mice using Phaseolus vulgaris leuco-agglutinin 
(PHA-L). Exp Brain Res 1991; 87: 159-172, doi: 10.1007/ BF00228517.

23. Sherman SM, Guillery RW. Distinct functions for direct and transthalamic corticocortical connections. I Neurophysiol 2011; 106: 1068-1077, doi: 10.1152/jn.00429.2011.

24. Coogan TA, Burkhalter A. Hierarchical organization of areas in rat visual cortex. J Neurosci 1993; 13: 3749-3772.

25. Hellwig B. A quantitative analysis of the local connectivity between pyramidal neurons in layers $2 / 3$ of the rat visual cortex. Biol Cybern 2000; 82: 111-121, doi: 10.1007/PL00007964.

26. Ahmed B, Anderson JC, Douglas RJ, Martin KA, Nelson JC. Polyneuronal innervation of spiny stellate neurons in cat visual cortex. J Comp Neurol 1994; 341: 39-49, doi: 10.1002/ cne.903410105.

27. McGuire BA, Hornung JP, Gilbert CD, Wiesel TN. Patterns of synaptic input to layer 4 of cat striate cortex. J Neurosci 1984; 4: 3021-3033.

28. Anderson JC, Martin KA. Does bouton morphology optimize axon length? Nat Neurosci 2001; 4: 1166-1167, doi: 10.1038/nn772.

29. Pouchelon G, Frangeul L, Rijli FM, Jabaudon D. Patterning of pre-thalamic somatosensory pathways. Eur J Neurosci 2012; 35: 1533-1539, doi: 10.1111/j.1460-9568.2012.08059.x.

30. Yu C, Derdikman D, Haidarliu S, Ahissar E. Parallel thalamic pathways for whisking and touch signals in the rat. PLOS Biol 2006; 4: e124, doi: 10.1371/journal.pbio.0040124.

31. Simpson KL, Waterhouse BD, Lin RC. Origin, distribution, and morphology of galaninergic fibers in the rodent trigeminal system. J Comp Neurol 1999; 411: 524-534, doi: 10.1002/(SICI)1096-9861(19990830)411:3<524::AIDCNE13 > 3.0.CO;2-X

32. Petrof I, Sherman SM. Functional significance of synaptic terminal size in glutamatergic sensory pathways in thalamus and cortex. J Physiol 2013; 591: 3125-3131, doi: 10.1113/ jphysiol.2012.247619.

33. Tarczy-Hornoch K, Martin KA, Stratford KJ, Jack JJ. Intracortical excitation of spiny neurons in layer 4 of cat striate cortex in vitro. Cereb Cortex 1999; 9: 833-843, doi: 10.1093/cercor/9.8.833.

34. De Paola V, Holtmaat A, Knott G, Song S, Wilbrecht L, Caroni $\mathrm{P}$, et al. Cell type-specific structural plasticity of axonal branches and boutons in the adult neocortex. Neuron 2006; 49: 861-875, doi: 10.1016/j.neuron.2006.02.017.

35. Stettler DD, Yamahachi H, Li W, Denk W, Gilbert CD. Axons and synaptic boutons are highly dynamic in adult visual cortex. Neuron 2006; 49: 877-887, doi: 10.1016/j.neuron.2006.02.018.

36. Bahia CP, Houzel JC, Picanco-Diniz CW, Pereira A Jr. Spatiotemporal distribution of proteoglycans in the developing rat's barrel field and the effects of early deafferentation. J Comp Neurol 2008; 510: 145-157, doi: 10.1002/cne.21781.

37. Shu SY, Ju G, Fan LZ. The glucose oxidase-DAB-nickel method in peroxidase histochemistry of the nervous system. Neurosci Lett 1988; 85: 169-171, doi: 10.1016/0304-3940 (88)90346-1.

38. Rocha EG, Santiago LF, Freire MA, Gomes-Leal W, Dias IA, Lent $\mathrm{R}$, et al. Callosal axon arbors in the limb representations of the somatosensory cortex (SI) in the agouti (Dasyprocta primnolopha). J Comp Neurol 2007; 500: 255-266, doi: 10.1002/cne.21167.

39. Schweitzer $L$, Renehan WE. The use of cluster analysis for cell typing. Brain Res Brain Res Protoc 1997; 1: 100-108, doi: 10.1016/S1385-299X(96)00014-1.

40. Steele GE, Weller RE. Qualitative and quantitative features of axons projecting from caudal to rostral inferior temporal cortex of squirrel monkeys. Vis Neurosci 1995; 12: 701-722, doi: 10.1017/S0952523800008981. 